INPLASY

PROTOCOL

To cite: Zhou et al. Chinese herbal medicine Wutou decoction for knee osteoarthritis: A protocol for systematic review and metaanalysis. Inplasy protocol

202090022. doi:

10.37766/inplasy2020.9.0022

Received: 05 September 2020

Published: 05 September 2020

Corresponding author:

Zhou Xing

a459378343@126.com

Author Affiliation:

Taizhou Hospital of Traditional

Chinese Medicine, Zhejiang

Province

Support: None.

Review Stage at time of this submission: The review has not yet started.

Conflicts of interest:

The authors declare no conflicts of interest.

\section{Chinese herbal medicine Wutou decoction for knee osteoarthritis: A protocol for systematic review and meta-analysis}

Zhou, $\mathrm{X}^{1}$; Xiang, KM²; Yuan, $\mathrm{XY}^{3}$.

Review question / Objective: The aim of this review is to evaluate the effectiveness and safety of Wutou decoction for knee osteoarthritis.

Condition being studied: Knee Osteoarthritis (KOA), known as a common clinical chronic and degenerative disease, which is more common in middle-aged and elderly people, especially in female patients. Joint cartilage degeneration and synovial lesions are are the characteristics of KOA. The main clinical manifestations are poor mobility, and pain, which contributed by the fascia, ligament and tendon attachment point, synovium, joint Capsules and subchondral bone etc. Advanced knee osteoarthritis may lead to disability, that harmful the quality of life of patients. At the stage of early and mid-term of KOA, conservative treatment is the main treatment, and surgical intervention is often used in the late period. NSAIDs are the first choice for the treatment of KOA, But long-term use of NSAIDs has a higher incidence of gastrointestinal adverse reactions and cardiovascular events.

INPLASY registration number: This protocol was registered with the International Platform of Registered Systematic Review and Meta-Analysis Protocols (INPLASY) on 05 September 2020 and was last updated on 05 September 2020 (registration number INPLASY202090022).

\section{INTRODUCTION}

Review question / Objective: The aim of this review is to evaluate the effectiveness and safety of Wutou decoction for knee osteoarthritis.
Condition being studied: Knee Osteoarthritis (KOA), known as a common clinical chronic and degenerative disease, which is more common in middle-aged and elderly people, especially in female patients. Joint cartilage degeneration and 
synovial lesions are are the characteristics of KOA. The main clinical manifestations are poor mobility, and pain, which contributed by the fascia, ligament and tendon attachment point, synovium, joint Capsules and subchondral bone etc. Advanced knee osteoarthritis may lead to disability, that harmful the quality of life of patients. At the stage of early and mid-term of KOA, conservative treatment is the main treatment, and surgical intervention is often used in the late period. NSAIDs are the first choice for the treatment of KOA, But longterm use of NSAIDs has a higher incidence of gastrointestinal adverse reactions and cardiovascular events.

\section{METHODS}

Search strategy: CNKI, Wanfang, VIP, CBM, PubMed, Embase and Cochrane Library databases were searched for this study.Take the subject terms combined with free words to search, take PubMed as an example:terms consist of disease (osteoarthritis, knee OR knee osteoarthritis OR knee osteoarthritides OR Osteoarthritis of Knee OR Osteoarthritis of the Knee) and intervention (Wutou decoction OR Wutou Tang OR modified Wutou decoction) and research types (randomized controlled trial OR controlled clinical trial OR random trials).

Participant or population: Patients were diagnosed with knee osteoarthritis and the study belongs to randomized controlled trial. Clinical results included clinical effectiveness and Visual analog scale (VAS). Experimental group must contain Wutou decoction or modified Wutou decoction. and control group was not limited except that. Otherwise, studies will be excluded if they cannot meet the inclusion criteria.

Intervention: Intervention of the experimental group is Wutou decoction or modified Wutou decoction.There are no restrictions on the way of administration, dosage and treatment period.
Comparator: The control group have other treatment methods different from Wutou decoction or modified Wutou decoction.

Study designs to be included: Only randomized controlled trials will be included in this study.

Eligibility criteria: Randomized clinical trials will be included irrespective of blinding, publication status or language.

Information sources: We will search articles in seven electronic database including: CNKI, Wanfang, VIP, CBM, PubMed, Embase and Cochrane Library databases. All the publications, with no time restrictions, will be searched without any restriction of countries or article type. Reference list of all selected articles will independently screened to identify additional studies left out in the initial sea.

Main outcome(s): The primary outcome is Visual analog scale(VAS).

Additional outcome(s): The secondary outcome are clinical effectiveness and the incidence of adverse reaction.

Data management: (1)NoteExpress and Excel software will be used to extract data, and the content will be stored in electronic chart. (2)Different researchers will separately screen the titles and abstracts of records acquiaed potential eligibility which comes from the electronic databases. Full texts screening and data extraction will be conducted afterwards independently. Any disagreement will be resolved by discussion until consensus is reached or by consulting a third author.In this step, we will use NoteExpress. (3) The following data will be extracted: author, year of publication, country, interventions of experimental groups and control groups, time point, outcome measures, age of patients, total number of people included in the study, patients' basic information, etc. Different researchers will separately extract data. Any disagreement regarding data extraction will be will be resolved by discussion until consensus is reached or by 
consulting a third author. In this step, we will use Excel.

Quality assessment / Risk of bias analysis: Two reviewers will independently assesses the quality of the selected studies according to the Cochrane Collaboration's tool for randomized controlled trials. Items will be evaluated in three categories: Low risk of bias, unclear bias and high risk of bias. The following characteristics will be evaluated: random sequence generation (selection Bias), allocation concealment (selection bias), blinding of participants and personnel (performance bias), incomplete outcome data (attrition bias), selective reporting (reporting bias)and other biases. Results from these questions will be graphed and assessed using Review Manager 5.4. The results will be presented in the form of a graph, and will be independently evaluated by two researchers. If there are differences of opinion, they will be discussed with the third researcher.

Strategy of data synthesis: Statistical analysis will be conducted using RevMan 5.4 software.For continuous data, will be used mean difference (MD) as the effect indicator with $95 \%$ confidence interval, and dichotomous data will be calculated as risk ratio (RR) or odds ratio (OR)as the effect index with $95 \%$ confidence interval. If the studies with no statistical homogeneity, the fixed-effect model can be used for analysis; if the studies with significant statistical heterogeneity, random effects model analysis will be used.

Subgroup analysis: We will consider subgroups analysis intervention of the experimental group.

Sensibility analysis: Through sensitivity analysis assess the source of heterogeneity, by excluding low-quality studies, or by excluding one of the included studies in turn, if there is no significant change in the heterogeneity, the results are robust, otherwise, the excluded study is heterogeneous originate.
Country(ies) involved: China.

Other relevant information: None.

Keywords: systematic review; protocol; Wutou decoction; knee osteoarthritis; randomized controlled trial.

Dissemination plans: We plan to publish a systematic review based on this protocol.

Contributions of each author:

Author 1 - Zhou Xing - Drafted and improved the manuscript.

Author 2 - Xiang Ke-meng - Revise this protocol; search strategy; analysis of results.

Author 3 - Yuan Xiang-yao - data collection.

Language: No restriction on language. 